\title{
Obesity Prevention In Infants, Children And Adults: An Interdisciplinary Approach
}

Shari McMahan, PhD, MCHES, California State University Fullerton, USA

Graciela Amaya, MS, California State University Fullerton, USA

\begin{abstract}
The Center for the Promotion of Healthy Lifestyles and Obesity Prevention, housed in the College of Health and Human Development, at California State University Fullerton promotes interdisciplinary research and community outreach in areas related to children's health and weight management. Three research projects presented below offer a comprehensive multi-level approach that guides us in developing current and future programs that are effective for addressing obesity in infants, children, and adults. These projects range from creating and testing a telenovela, designing a healthy eating program emphasizing breakfast nutrition and physical activity, and finally looking at the role of the environment in terms of commute times and obesity patterns.
\end{abstract}

Keywords: Obesity Prevention; Environment; Health Education

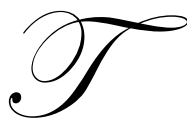

he dramatic increase in childhood obesity over the past decade has become one of the country's most urgent public health concerns. Recent reports from the Centers for Disease Control estimate that one in three U.S. children will become diabetic, largely due to obesity and inactivity, with the odds for Hispanic children being closer to one in two. Because the nature of this problem is so complex and intractable, its solution will require a interdisciplinary approach to combat this important public health issue (Centers for Disease Control and Prevention, 2011).

The Center for the Promotion of Healthy Lifestyles and Obesity Prevention, housed in the College of Health and Human Development, at California State University Fullerton promotes interdisciplinary research and community outreach in areas related to health and weight management. The Center facilitates interaction among multiple entities concerned with the rise in children's obesity including university faculty, schools, community agencies, and policy makers.

The Center's mission is to:

- work with families, schools and other community agencies to develop and test model health promotion programs and interventions;

- $\quad$ assess and evaluate existing programs and policies;

- identify theoretical models and environments that can explain and influence behavior patterns across different high-risk groups.

As such, the mission and goals of the Center reflect the needs of the community by providing education and outreach to children and their families regarding healthy lifestyles and prevention of obesity.

The interdisciplinary center at California State University, Fullerton builds upon current research, evaluation and intervention research of faculty and community collaborations in Orange County, focusing particularly on the Latino population. The following three projects offer a comprehensive multi-level approach that will inform the design and implementation of current and future programs that are effective for addressing obesity in infants, children, and adults. 
- Project \#1: Promoting Breastfeeding to Latinas: "an Evaluation of a Telenovela"

- $\quad$ Project \#2: "Kick Start Your Day"

- $\quad$ Project \#3: Commuting Behaviors, Obesity Risk, and the Built Environment

\section{Project 1: Stopping Childhood Obesity Before it Starts: Evaluation of a Telenovela Promoting Breastfeeding to Latinas}

Evidence suggests that breastfeeding and breastfeeding for longer durations protects against childhood obesity. Breastfed infants have lower rates of diabetes (DHHS, 2000). Current CDC estimates say 1 in 3 children will become diabetic, with the odds of Latino children being closer to 1 in 2 . Despite initiatives such as Healthy People 2010, only $64 \%$ of mothers breastfed during early postpartum in 1998, with $29 \%$ breastfeeding at 6 months and $16 \%$ at 1 year (DHHS, 2000). These rates are even lower for low-income women. In California, rates among low-income women vary. At the Women Infant Children program (WIC) the breastfeeding rate is only $10.1 \%$. Of particular concern is the low rate among minority participants. Although most U.S. Latinas recognize breastfeeding as superior, they face many barriers (Wood, Sasonoff, and Beal, 1998), including: embarrassment, difficulties with logistics, difficulties when working outside the home, and perceived lack of social support. While education, peer support, and training of health care professionals help, the challenge exists for a standardized, culturally sensitive intervention targeting the needs of minority women to breastfeed and breastfeed for longer durations. Phase I of this project included development of a 7-minute, Spanish-language telenovela (soap-opera themed, entertainmenteducation video) was developed as a culturally sensitive communication intervention to target Latinas. Phase 2 evaluated the telenovela intervention "Un Regalo Para El Babe" (A Gift for Baby) for its efficacy in increasing knowledge of the benefits of breastfeeding and promoting favorable attitudes and behavioral intention in regard to breastfeeding. A randomized control trial was used to evaluate the effectiveness of the entertainment education (EE) video among breastfeeding pregnant women attending prenatal health workshops at a WIC facility in Santa Ana, California. Participants were administered questionnaires at three time points (baseline, post, and follow-up). Questionnaire items included sections on: demographics, breastfeeding history, breastfeeding comfort level, breastfeeding barriers, and breastfeeding attitude. A control group received a regular breastfeeding education program. The intervention group received the regular breastfeeding education program with the video (entertainment education) intervention.

One hundred fifty two women who participated at the baseline of the study, 141 post intervention, and 85 participants completed the final questionnaire three-six months after their babies were delivered. Three significant short-term outcomes resulted from this study: 1)an increased comfort level of engaging in conversations about the benefits of breastfeeding with a family member or friend, 2) an increased belief that breastfeeding can also prevent babies from suffering from obesity and 3) an increased understanding that it is not fine if you stop breastfeeding after a month or two.

\section{Project 2: "KIck Start Your Day": A Program to Educate Hispanic Families about Healthy Eating and Exercise Using an Innovative Strategy}

The prevalence of obesity and overweight in the United States continues to rise. Numerous factors such as consumption of high fat foods, large portion sizes and a sedentary lifestyle, contribute to weight gain and obesity (Ogden, Flegal, Carrol, \& Johnson, 2002). Ogden et al. (2002) stated that overweight and obesity are related to dietary intake and physical activity which are both influenced by physical environment, social, economic and cultural factors.

Results from the Behavioral Risk Factor Surveillance System suggest that the prevalence of obesity in childhood is increasing by an accelerating rate (Strauss, \& Pollack, 2001).

In an attempt to address the increased prevalence of obesity in the Mexican American community, an understanding of the specific nutritional needs, culture, beliefs and health concerns of this population are necessary. Among the Mexican American community, there are many obstacles, such as limited resources, literacy, child care, health beliefs, language, culture, time, transportation and transience that need to be addressed for any form of prevention interventions to be successful (Taylor, et al., 2000). Intervention programs based on sound theory that 
consider both the health problem and the population for which the intervention is intended, will be more effective in bringing about change. The purpose of this project is to educate Hispanic families about healthy eating and exercise using an innovative strategy called "Kick Start Your Day".

"Kick Start Your Day" was a six week, community trial study to implement and test the effectiveness of a low-literacy, culturally appropriate community education intervention to incorporate a healthy breakfast and physical activity into a morning routine among a total of 20-25 Hispanic families with children between the ages of 6 and 12. Families were taught the importance of eating nutrient dense foods, avoiding nutrient -lacking, high calorie, high glycemic foods and exercising. The goals for the intervention were as follows:

1. Educate families on the importance of a healthy start (eating and exercise) in the morning.

2. Provide nutritious breakfast foods to families participating in the program.

3. Teach parents what a healthy portion size is and how to read/evaluate nutrition labels.

4. Engage children in brief exercise sessions to demonstrate/teach fun and easy activities.

From pre-test to post-test, the intervention group increased their belief that breakfast helps you pay attention and helps regulate moods. Breakfast changes (e.g. egg consumption) increased from baseline to the $3^{\text {rd }}$ assessment period (follow-up) for the intervention group relative to the control group. There were no significant changes in weight for either group. The intervention group improved their understanding of sodium content on nutrition labels. Participants in the intervention group also learned more about the glycemic index (what it is, and which foods are low glycemic). The kids liked the exercise components the most during their time in the program. Nearly all parents reported that their child showed them something new they learned.

\section{Project 3: Commuting Behaviors, Obesity Risk, and the Built Environment}

Obesity and overweight are now common in American society. College students are at particular risk for gaining weight. The urban environment, because of poor land-use planning, forces college students to become more automobile dependent. Automobile dependency and increased drive time can take away from a more active lifestyle, possibly putting one at risk for adverse health outcomes. A recent publication consisting of 10,878 residents of the 13 county Atlanta regions found time spent in a car as a passenger or driver was positively associated with obesity, and an additional 60 minutes per day in the car translated into an additional 6\% odd of being obese (Frank, Martin, Andresen, \& Schmid, 2004).

A questionnaire regarding commuting patterns, health behaviors and reflections of youth behaviors was created based on review of the existing literature. Students at California State University Fullerton $(n=496)$ enrolled in Personal Health 101 during Fall semester were invited to participate. Course instructors permitted students to complete the 10-15 minute questionnaire during the first 20 minutes of class. Body Mass Index was collected as part of the exercise physiological lab protocol ( $\mathrm{N}=361)$. All 496 participants were analyzed except when the outcome was measured with body mass index. Controlling for covariates, the amount of time spent commuting in one's car has an effect on weight. Those who report a drive time of 16 minutes or greater are $64 \%$ more likely to be overweight then those who reported a drive time of 15 minutes or less. Students who reported a drive time of 16 minutes or greater are more likely to consume food in their vehicles $(\mathrm{p}<0.013)$. Eating in one's car is highly correlated with fast food consumption $(\mathrm{P}<0.009)$. Most participants perceive commuting as having a slightly or somewhat negative effect on their physical activity (64.4\%). While $7.9 \%$ of participants felt that commuting very much affected their physical activity. Most students (60.7\%) perceive commuting as having a slightly or somewhat negative effect on their diet and $10.5 \%$ reported that commuting very much so effects their diet.

The research projects illustrated above demonstrate the importance of an interdisciplinary approach to prevent obesity. Successful programs must have interventions that target not only the individual level but family, community (including schools and after-school programs), environmental and policy levels to be effective. 


\section{AUTHOR INFORMATION}

Shari McMahan, PhD, MCHES, is Dean of the College of Health and Human Development at California State University Fullerton and Director for the Center for the Promotion of Healthy Lifestyles and Obesity Prevention. Her research utilizes a social ecological approach in the prevention of obesity and has been awarded $\$ 1,000,000.00$ in research grants to promote healthy lifestyles. Dr. McMahan is a Masters Level prepared Certified Health Education Specialist and past Vice President of Health and Research divisions for the Southwest District of the American Alliance for Health, Physical Education, Recreation and Dance. E-mail: smcmahan@fullerton.edu. Corresponding author.

Graciela Amaya, MS, is an Assistant for the Health Promotion Research Institute, Center for Successful Aging, and the Center for Healthy Lifestyles and Obesity Prevention at California State University, Fullerton. Her research interests are working with the community health needs of older adults and children to promote health and wellbeing. She has over 10 years experience assisting faculty in the departments of Kinesiology and Health Science with research grants ranging from obesity research, fall prevention, fibromyalgia, and cancer related research. Email: gamaya@fullerton.edu

\section{REFERENCES}

1. Centers for Disease Control and Prevention (2011). National Center for Chronic Disease Prevention and Health Promotion. Obesity Halting the Epidemic by Making Health Easier. At a glance 2011. Accessed August 15, 2011. http://www.cdc.gov/chronicdisease/resources/publications/AAG/obesity.htm.

2. Frank LD, Andresen MA, Schmid TL. (2004). Obesity relationships with community design, physical activity, and time spent in cars. Am J Prev Med. 2004, 27(2):87-96.

3. Ogden, C, L., Flegal, K.M., Carrol, M.D., \& Johnson, C.L. (2002). Prevalence and trends in overweight among US children and adolescents, 1999-2000. JAMA: The Journal of the American Medical Association, 288 (14), 1728-1732.

4. Strauss, R. \& Pollack, H. (2001). Epidemic increase in childhood overweight, 1986-1998. JAMA: The Journal of the American Medical Association, 286 (22), 2845-2848.

5. Taylor, T., Serrano, E., Anderson, J., \& Kendall, P. (2000). Knowledge, skills, and behavior improvements on peer educators and low-income Hispanic participants after a stage of change-based bilingual nutrition education program. Journal of Community Health, 25, 241-262.

6. US Department of Health and Human Services (2000). Office on Women's Health. HHS Blueprint for Action on Breastfeeding. Washington D.C.

7. Wolf, J.H. (2003). Low breastfeeding rates and Public health in the United States. American Journal of Public Health, 93, 2000-2010.

8. Wood, S. P., Sasonoff, K. M., \& Beal, J. A. (1998). What's happening breast-feeding attitudes and practices of latino women a descriptive study. Journal of the American Academy of Nurse Practitioners, 10, 253260. 